\title{
DESIGN, CONSTRUCTION AND EXPERIMENTAL VERIFICATION OF MAGNETORHEOLOGICAL BRAKE
}

This article deals with the principle, design and verification of the magnetorheological brake. Physical principles, possible designs and advantages of magnetorheological brakes are presented. A design is chosen, simulated and device is built. Simulations are compared with results obtained from measurements on the device, suitable magnetorheological fluid is chosen and torque characteristics of the brake are experimentally verified.

Keywords: Controlled brake, dynamical characteristics, magnetorheological fluid.

\section{Introduction}

Classical brakes possess several disadvantages. Friction of solid mechanical parts wears out the device, the need of hydraulic parts increases space requirements and the control of such brakes is difficult due to the passivity of conventional systems and due to the need of active elements. Magnetorheological brakes offer solution to these problems: they contain no solid to solid part joints, allow continuous changes of deceleration and they are direct electromechanical converters, which makes their control simple. Presentday industry uses magnetorheological brakes in several applications and many designs of magnetorheological brakes can be found nowadays $[1,2]$.

\section{Properties of magnetorheological fluids}

Magnetorheological fluids are fluids able to change their viscosity depending on the applied magnetic field. These fluids are thus an example of so called smart materials. They consist of micron sized metallic particles dispersed in a carrier liquid, most often oil.
Without an external magnetic field, these particles are randomly scattered in carrier oil and do not influence the viscosity of the whole fluid. If an external magnetic field is applied, particles are organized in the direction of the field and resist possible movements thus increasing the viscosity of overall liquid, as seen in Fig. 1.

Highest viscosity change is measured in the movement direction perpendicular to the direction of the applied magnetic field. Devices working on the change of magnetorheological fluid viscosity principle are thus often geometrically designed to work in such a way.

Magnetorheological fluids are nonconductive and the size of their ferromagnetic particles is in micrometers. Such a small size means that eddy currents are not present in the fluid or particles, which makes their magnetization characteristics static [3].

\section{Used Magnetorheological Fluid}

Magnetorheological fluid MRHCCS4-B obtained from Liquids Research Ltc. was used as the braking liquid of proposed device.
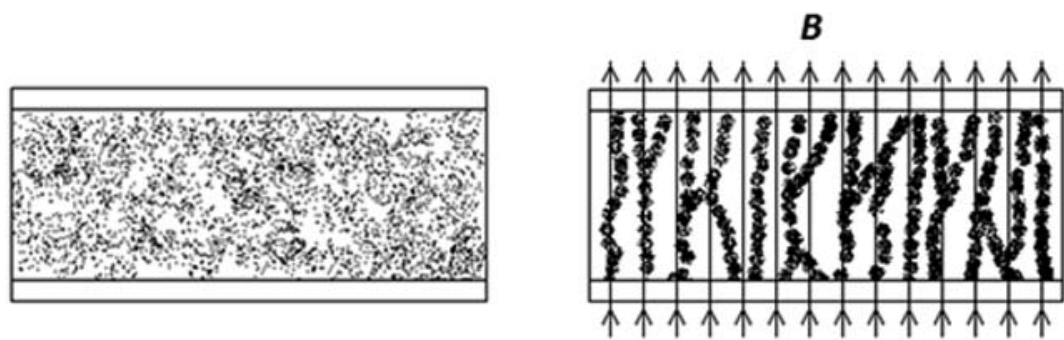

Fig. 1 Magnetic fluids viscosity change: Left - Magnetorheological fluid without external magnetic field; Right - Magnetic particles are organized in the direction of the applied magnetic field

\footnotetext{
* Petr Polcar

Faculty of Electrical Engineering, University of West Bohemia, Pilsen, Czech Republic, E-mail: paladin@kte.zcu.cz
} 
Its magnetic properties were determined using our method for magnetic properties measurement mentioned in [4]. The relative permeability in the linear part of magnetization characteristics is $\mu_{r}=1.61$. The change of viscosity depending on the perpendicular applied magnetic field was obtained from the University of Timisoara measurements [5]. Saturated magnetization of this fluid is $750 \mathrm{mT}$.

\section{Brake Design}

The physical principle of the magnetorheological brake design can be seen in Fig. 2. The brake disc is rotating in a gap of a magnetic circuit filled with the magnetorheological fluid. If coils are powered, the resulting magnetic field is led through the magnetic circuit and influences the fluid in its gap. By changing the viscosity of the fluid, the braking of the whole system can be continually controlled. No direct connection of metal parts is present, and braking is achieved by viscous losses in the fluid.

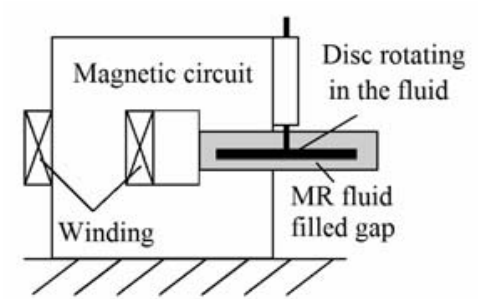

Fig. 2 Magnetorheological brake principle scheme

Most mechanically stressed part of the brake is the connection of the disc and the shaft. These parts must be properly fixed in order to withstand the resulting torque. The material the disc is made of has impact on the brake principle - if it is made of conductive material, the device works as an eddy current brake as well because of eddy currents inducted into the moving conductive material [6]. These currents generate magnetic field and forces in the opposite direction to the movement. Resulting drag torque is then given by the superposition of the drag torque generated by eddy currents and the drag torque generated by viscous losses. If the material is nonconductive, the device works as a pure magnetorheological brake, but nonconductive materials possess worse mechanical properties.

Significant disadvantage of this braking system is the initial viscosity of the fluid without the presence of an external magnetic field. The rotating disc is braked even without the applied magnetic field. Resulting energy is changed into heat and warms used fluid, which results in cooling requirement.

\section{Mathematical Model of the Magnetic Field and Experimental Verification}

Magnetic field in range $B=0 \sim 400 \mathrm{mT}$ is needed in the brake gap to achieve demanded viscosity change of the magnetorheolog- ical fluid. Magnetic circuit was made and the problem was simulated to properly design the number of coils and to choose suitable power source for the brake coils. Magnetic field in the area of the magnetic fluid filled gap is observed. Because of known magnetic properties of the fluid $\left(\mu_{r}=1.61\right.$ in the linear part of the magnetization characteristics and the saturation magnetization of $750 \mathrm{mT}$ ) the problem can be treated as non coupled, the changes in magnetic fluid do not influence resulting magnetic field, and linear, the device operates in the linear part of the magnetization curve. The value of this field was computed using the mathematical model respecting the coil filling coefficient, then measured on the experimental device and results were compared.

Mathematical model of the problem was created using the FEM application Agros2D. The magnetic vector potential equation was solved in order to acquire the distribution of the magnetic field.

$$
\operatorname{rot} \frac{1}{\mu} \operatorname{rot} A=J_{e x t}
$$

The value of the magnetic field $B$ can be easily obtained from the formula

$$
B=\operatorname{rot} A
$$

The convergence of results was observed. The mathematical model with 7689 nodes and 3216 elements built in Agros2D software was found accurate and quick enough. Second order elements were used to ensure higher accuracy and faster computing of the model. Distribution of the resulting magnetic field can be seen in Fig. 3.

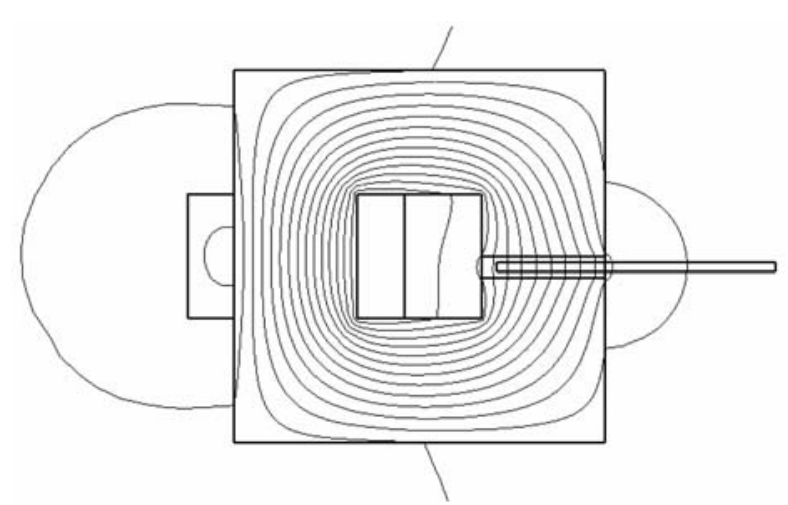

Fig. 3 Distribution of the magnetic field obtained from the numerical simulation of the brake in Agros2D software

Resulting magnetic field for different values of winding current was compared with values obtained by measuring on the magnetic circuit using the Elimag MP-1 fluxmeter with hall probe injected into the magnetic fluid filled gap. Results confirming the assumption that the device works in the linear part of the magnetization characteristics can be seen in Fig. 4. 


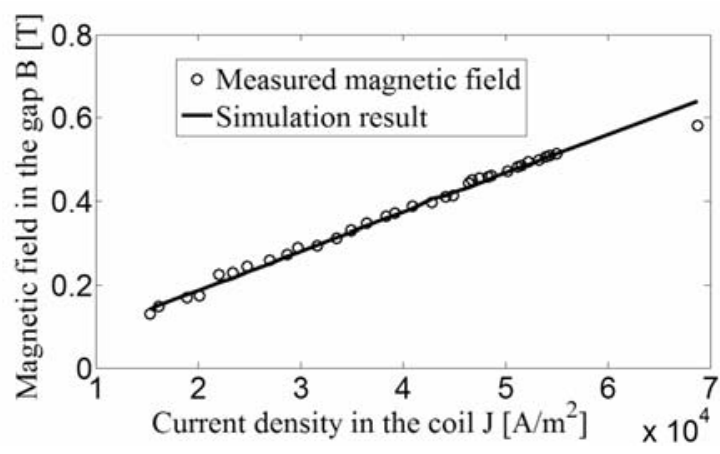

Fig. 4 Results of the magnetic field in the brake gap mathematical modeling compared with measured results

\section{Experimental Device}

An experimental device of magnetorheological brake was constructed and can be seen in Fig. 5. The magnetorheological fluid container is open to ensure better maintenance of the device and easy injection of measurement probes. This container would be sealed in a real application. Magnetorheological fluid MRHCCS4$\mathrm{B}$ was used to fill it. The coils are powered with direct current so skin-effect is not present and construction steel is sufficient for the magnetic circuit material, there is no need for transformer plates. Current density is achieved by powering 500 coils made of $0.6 \mathrm{~mm}$ copper wire with values of direct current from up to $3 \mathrm{~A}$ DC power source. The same values of current density can be achieved using more coils and fewer currents as well. The disc shaft and the disc itself are made of aluminum in order to obtain higher mechanical strength of disc to shaft connection.

The operation of the fluid in the device can be seen in Fig. 6 .

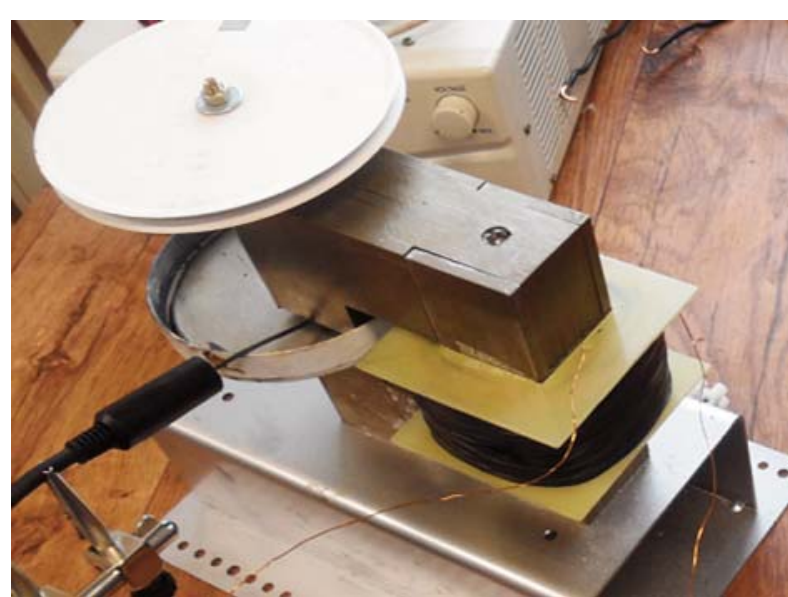

Fig. 5 Experimental magnetorheological brake with hall probe measuring the magnetic field in the brake chamber

\section{Drag Torque of the Magnetorheological Brake}

The viscous drag torque of a disc rotating in a fluid depends on the fluid viscosity $\eta$, revolution of the disc $\omega$ and the geometrical dimensions of the disc. This torque can be counted from the acquired values of the magnetic field and viscosity-magnetic fielddisc revolution characteristics of the used fluid MRHCCS4-B [5]. As can be seen, the drag torque is present even when the fluid is not affected by magnetic field, the magnetorheological fluid possesses an initial viscosity causing viscous losses. When the viscosity rises, the drag torque changes as well. The total drag torque can be thus expressed as the superposition of drag torque caused by the initial viscosity, the drag torque caused by the increase of the viscosity and the drag torque caused by the eddy currents braking.
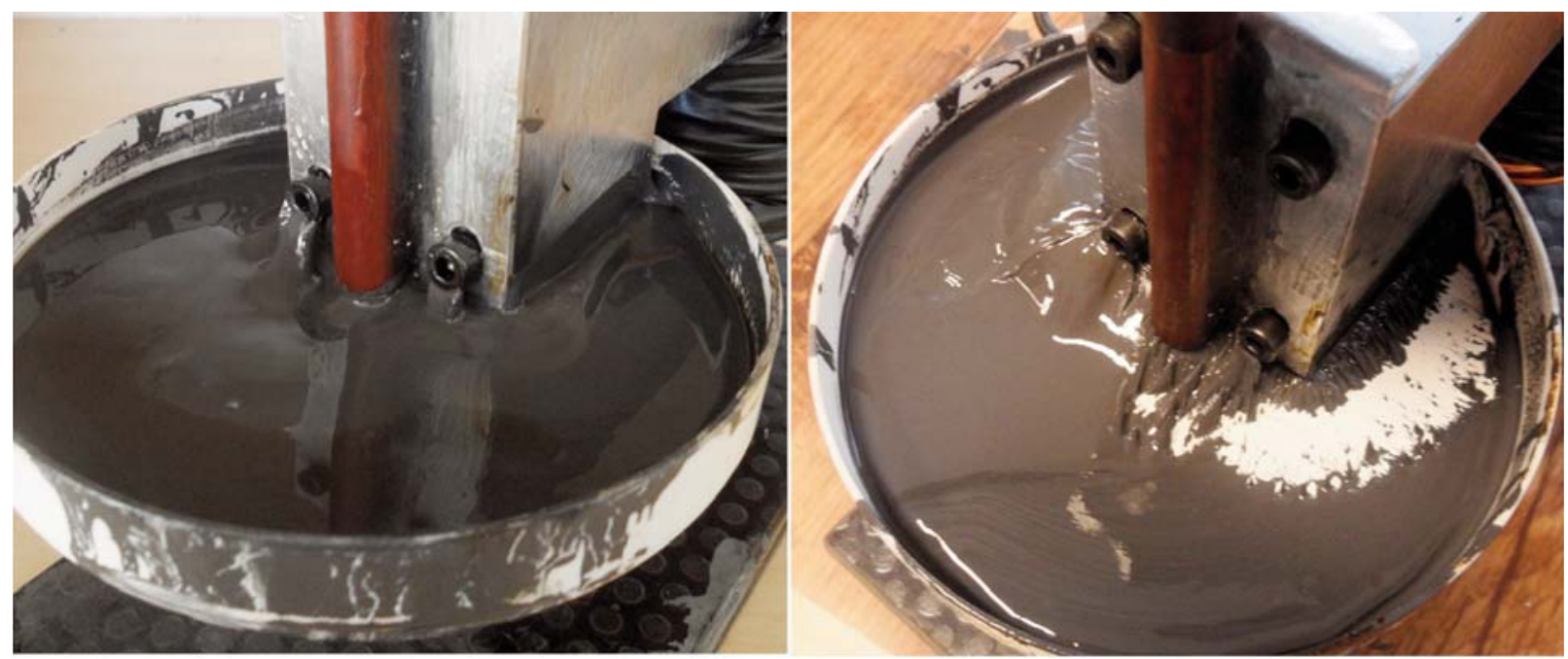

Fig. 6 Magnetorheological brake influences the viscosity of the fluid in the gap: Left - Coils are not powered; Right - Coils are powered with current density of $6 \cdot 10^{4} \mathrm{~A} / \mathrm{m}^{2}$ 


$$
M=M_{\text {rheo }(I=0)}+M_{\text {rheo }}(I)+M_{\text {eddy }}
$$

The drag torque Meddy caused by eddy currents induced into the disc is given by the magnetic field and the square of the disc rotation and can be easily deduced from the elemental relations given by the electromagnetic field theory (see e.g. [7]). Because of the low values of the magnetic field in the brake gap (in order of hundreds of $\mathrm{mT}$ ), the value of this torque is several orders lower than the values of the torques given by viscous losses and can be neglected. This assumption was experimentally verified by measuring the drag torque of the brake with an aluminum disc without the presence of the magnetorheological fluid.

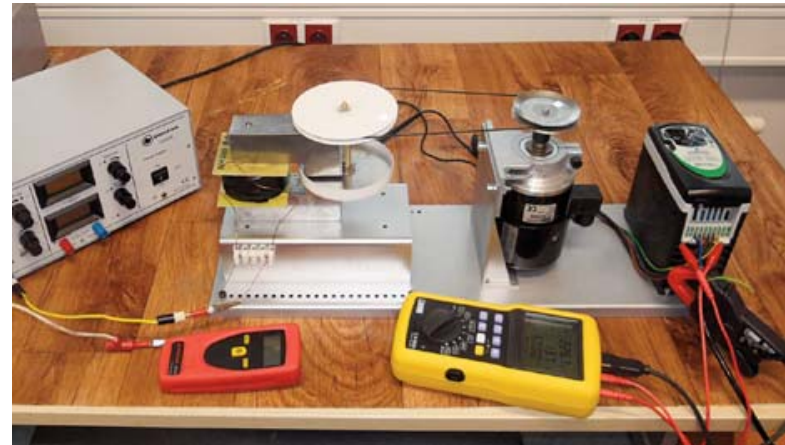

Fig. 7 The setup for measuring the dynamical drag torque of the magnetorheological brake

The dynamical drag torque characteristics of the device were measured using the experimental setup that can be seen in Fig. 7 .

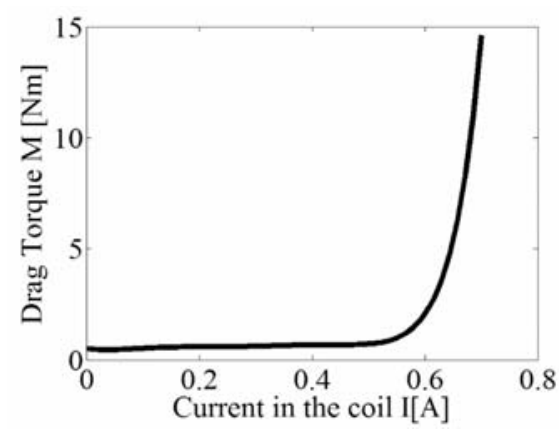

Fig. 8 Measured drag torque as a function of current powering the brake coil
The experimental brake was driven by an asynchronous machine FT4C52S. The mechanical revolution of the brake disc was measured with tachometer and the active power supporting the asynchronous machine was observed using a power network analyzer. The relation between the power and the disc revolution is given by:

$$
P_{\kappa}=M_{\omega} .
$$

Where $P$ stands for the active power, $\kappa$ represents the machine efficiency and $\omega$ is the revolution of the disc. The drag torque characteristics depending on the current powering the brake coils can be seen in Fig. 8 .

Drag torque $\operatorname{Mrheo}(I=0)$ caused by the initial viscosity of the magnetorheological fluid is not present in the characteristics, the graph shows the increase in the drag torque caused by the viscosity change of the magnetorheological fluid. When driven by the used motor, the revolution of the disc dropped under $\omega=1 \mathrm{~s}^{-1}$ when powering the coil with current higher than $I=0.7 \mathrm{~A}$. The disc stopped rotating when the coil was powered with the current of $I=1 \mathrm{~A}$

\section{Conclusion}

A design of a magnetorheological brake was presented and an experimental mechanism was built. This brake has several advantages: no direct solid to solid part connection causing negligible mechanical wear, direct electric control and low space requirements. Current density in order of $J=10^{4} \mathrm{~A} / \mathrm{m}^{2}$ is enough to create such a viscosity change in the MRHCCS4-B fluid to stop the movement of the device driven by the used motor. Such values of current densities are quite easy to achieve, it was accomplished by 500 coils powered with $1 \mathrm{~A}$ in our case. The prime disadvantage of this brake design is the passive braking caused by viscous losses in injected fluid even without the applied magnetic field that predetermines the brake for the use in relatively slow running devices. Optimizing the magnetic circuit to decrease power requirements of the device and acquiring drag torque characteristics of the brake with different types of magnetorheological fluids is the theme of our future research in this field of interest.

\section{Acknowledgment}

This work was supported by the specific research project of the University of West Bohemia SGS-2012-039.

\section{References}

[1] LI, W. H., DU, W. H.: Design and Experimental Evaluation of a Magnetorheological Brake. Intern. J. of Advanced Manufacturing Technology, 21(7), pp. 508-515, 2003.

[2] WANG, J., MENG, G.: Magnetorheological Fluid Devices: Principles, Characteristics and Applications in Mechanical Engineering. Proc. of the Institution of Mechanical Engineers Part L: J. of Materials: Design and Applications, 215(3), pp. 165-174, 2001. 
[3] MAYER, D., POLCAR, P. A Novel Approach to Measurement of Permeability of Magnetic Fluids. Przeglad Elektrotechniczny, 88(7 B), 2012, pp. 229-231.

[4] ODENBACH, S.: Ferrofluids - Magnetically Controlled Suspensions, Colloids and Surfaces, Physicochemical and Engineering Aspects, 2003, pp. 171-178.

[5] BALAN, C., BROBOANA, D., GHEORGHIU, E., VEKAS, L.: Rheological Characterization of Complex Fluids in Electro-magnetic Fields, J. of Non-Newtonian Fluid Mechanics, vol. 154, No. 1, September 2008, pp. 22-30, ISSN 0377-0257.

[6] JANOUSEK, L., MAREK, T., GOMBARSKA, D.: Eddy Current Non-destructive Evaluation of Conductive Materials. Communications - Scientific Letters of the University of Zilina, 8(1), pp. 29-33, 2006.

[7] DOLEZEL, I., KARBAN. P., SOLIN, P.: Integral Methods in Low-frequency Electromagnetics. Hoboken : John Wiley \& Sons, 2009. p. 388, ISBN 978-0-470-19550-5. 